\title{
Presynaptic Striatal Dopaminergic Function in Atypical Parkinsonism: A Metaanalysis of Imaging Studies
}

\author{
Valtteri Kaasinen $^{1-3}$, Tuomas Kankare ${ }^{2}$, Juho Joutsa ${ }^{1,2}$, and Tero Vahlberg ${ }^{4}$ \\ ${ }^{1}$ Division of Clinical Neurosciences, Turku University Hospital, Turku, Finland; ${ }^{2}$ Department of Neurology, University of Turku, \\ Turku, Finland; ${ }^{3}$ Turku PET Centre, University of Turku, Turku, Finland; and ${ }^{4}$ Department of Clinical Medicine, Biostatistics, \\ University of Turku, Turku, Finland
}

\begin{abstract}
Multiple-system atrophy (MSA), progressive supranuclear palsy (PSP), and corticobasal syndrome (CBS) have signs and symptoms overlapping those of Parkinson disease (PD), complicating their clinical diagnosis. Although presynaptic dopaminergic brain imaging with PET and SPECT is clinically widely used for patients with suspected $\mathrm{PD}$, the benefit of functional imaging in atypical parkinsonism syndromes remains unclear. We compared striatal presynaptic dopaminergic function in MSA parkinsonism variant (MSA-P), MSA cerebellar variant (MSA-C), PSP, CBS, and PD using combined quantitative data from all published studies. Methods: The PubMed database was searched from inception to August 2018 for the terms "dopamine" OR "dopaminergic" AND "PET" OR "SPECT" OR "SPET" and keywords related to PD, MSA, PSP, and CBS. In total, 1,711 publications were identified. PET or SPECT studies comparing patients with atypical parkinsonism to another diagnostic group (PD, MSA, PSP, or CBS) were included. Tracers for dopamine transporter (DAT), aromatic amino acid decarboxylase (AADC), or vesicular monoamine type 2 were investigated. Tracer binding data were extracted from the original articles. Heterogeneity of the data was examined using $I^{2}$ statistics, and a random-effects model was used to summarize data. Hedges $g$ was used as an estimator of effect size in group comparisons. Results are reported according to PRISMA guidelines. Results: Thirty-five studies (29 DAT, 6 AADC, no vesicular monoamine type 2 studies) with 356 MSA-P patients, 204 PSP patients, 79 CBS patients, and 62 MSA-C patients were included in the metaanalysis. Caudate nucleus and putamen DAT function was clearly lower in PSP than in PD (caudate: $34.1 \%$ difference, $g=-1.08$, $95 \%$ confidence interval $[\mathrm{Cl}]=-1.52$ to -0.64 ; putamen: $18.2 \%$, $g=-0.86,95 \% \mathrm{Cl}=-1.50$ to -0.21 ) and MSA-P (striatum: $31.4 \%$, $g=-0.70,95 \% \mathrm{Cl}=-1.21$ to -0.19$)$ and was clearly lower in MSA-P than in MSA-C (striatum: $46.0 \%, g=1.46,95 \% \mathrm{Cl}=0.23$ to 2.68 ). Although not significant because of limited data, aromatic L-AADC results paralleled the DAT findings. Conclusion: Striatal presynaptic DAT function is clearly lower in PSP patients than in PD and MSA-P patients and is clearly lower in MSA-P patients than in MSA-C patients.
\end{abstract}

Key Words: PET; SPECT; dopamine; parkinsonism; multiple system atrophy; progressive supranuclear palsy

J Nucl Med 2019; 60:1757-1763

DOI: 10.2967/jnumed.119.227140

\footnotetext{
Received Feb. 5, 2019; revision accepted Apr. 4, 2019.

For correspondence or reprints contact: Valtteri Kaasinen, Division of Clinical Neurosciences, Turku University Hospital, P.O. Box 52, FIN-20521, Turku, Finland.

E-mail: valtteri.kaasinen@tyks.fi

Published online Apr. 12, 2019.

COPYRIGHT @ 2019 by the Society of Nuclear Medicine and Molecular Imaging.
}

$\mathbf{M}$ ultiple-system atrophy (MSA), progressive supranuclear palsy (PSP), and corticobasal syndrome (CBS) have been termed atypical parkinsonian disorders and are characterized by a more rapid progression and poorer prognosis than the typical parkinsonian disorder, Parkinson disease (PD). Clinicopathologic studies have pointed out that the diagnostic accuracy of atypical parkinsonism is not optimal; these disorders are underdiagnosed, and many patients who carry a diagnosis of PD in fact have MSA, PSP, or CBS (1). The sensitivities of the MSA and PSP diagnoses are low, at $53 \%$ and $64 \%$, respectively, when diagnosed by general neurologists, and at $88 \%$ and $84 \%$, respectively, when diagnosed by movement disorder specialists $(2,3)$. Given that there are distinct proteinopathic disease mechanisms in different atypical parkinsonian syndromes and that there are active attempts to develop protein-specific therapies, biomarkers that could be used to improve diagnostic accuracy would be valuable.

Functional brain imaging with PET or SPECT enables the investigation of central neurotransmitter function at the system level in vivo. When PD patients are compared with healthy individuals via striatal dopaminergic PET/SPECT, the PD patients show a widespread presynaptic defect with practically no overlap with healthy controls (4). However, it remains unclear whether presynaptic dopamine imaging can be used in the differential diagnosis of atypical parkinsonism. Protein-specific tracers for tau and $\alpha$-synuclein hold promise as possible future diagnostic tools (5), but in the current clinical imaging of movement disorders, presynaptic dopaminergic imaging dominates the field. A major limitation in individual dopaminergic neuroimaging studies of atypical parkinsonism has been the small sample sizes, which have led to insufficient statistical power to make reliable clinical inferences.

A quantitative metaanalysis offers an opportunity to investigate a large number of small studies with improved power to detect differences. A previous metaanalysis using diagnostic odds ratios has suggested that presynaptic dopaminergic tracers cannot distinguish between PD and atypical parkinsonism (6). To investigate the role of presynaptic dopaminergic PET and SPECT in the diagnosis of atypical parkinsonism in more detail, we performed a metaanalysis of all available imaging data using regional binding values in each study.

\section{MATERIALS AND METHODS}

\section{Aims of Metaanalysis}

The primary aim of the metaanalysis was to investigate differences in striatal dopamine signaling as measured by PET/SPECT among atypical parkinsonism disorders as compared with PD. Ethics Committee 
approval was waived because this study did not involve any human participants or animals.

\section{Study Collection and Screening}

The Preferred Reporting Items for Systematic Reviews and MetaAnalyses (PRISMA) statement was followed (7). Studies for initial screening were identified through PubMed using a search query of key words related to parkinsonism disorders (Fig. 1). The final database search was conducted on August, 8, 2018. The initial screening and assessment for eligibility were performed by 2 investigators.

This study was an extension of a previously reported metaanalytical comparison between PD patients and healthy controls, using the same methodology (4). Studies were first screened on the basis of relevance in the title and abstract. Full-text articles were obtained and assessed if the articles were deemed relevant to the analysis, if their eligibility could not be determined from the title and abstract alone, or if the abstract was not available on PubMed. Dopaminergic synaptic mechanisms included in the analysis were aromatic L-amino acid decarboxylase (AADC), dopamine transporter (DAT), and vesicular monoamine transporter type 2 (VMAT2). Other criteria for inclusion are shown in Fig. 1. MSA studies that reported separate binding values for MSA parkinsonism variant (MSA-P) patients or MSA cerebellar variant (MSA-C) patients or

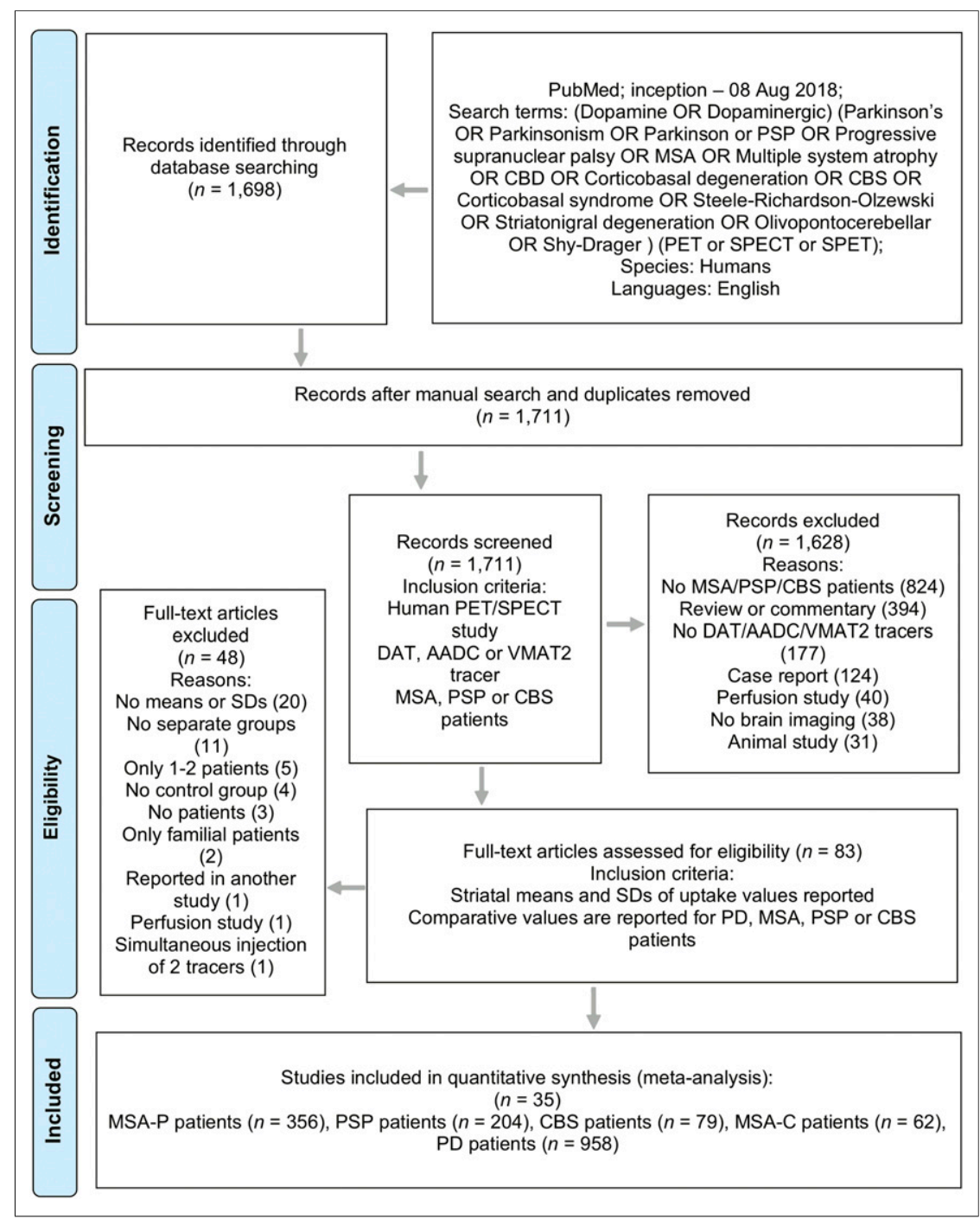

FIGURE 1. Flowchart of study inclusion and exclusion. $\mathrm{CBD}=$ corticobasal degeneration. both were included. Studies that did not specifically state MSA subgroups were considered to involve MSA-P, the more common subtype, if supported by the clinical features (i.e., the patients had parkinsonism). Studies that combined the binding values of MSA-P and MSA-C patients were excluded from the MSA comparisons. In the PSP studies, only Richardson syndrome phenotype patients were included.

\section{Data Extraction}

The study site, imaging method, and tracer binding values were extracted from the included studies. Variables extracted were the study year; first author name and institution; tracer compound and target; method of calculation for binding values; scanner model; sample sizes; mean and SD for age, duration of disease, motor and total Unified Parkinson's Disease Rating Scale (UPDRS) scores, and minimum/maximum Hoehn and Yahr scale scores; mean prescan carbidopa dose (mg) in the AADC studies; mean or range of dose of injected tracer $(\mathrm{MBq})$; scan duration ( $\mathrm{min}$ ); and binding values for each brain region analyzed. Hemispheric values were used to derive bilateral mean values if these were not provided in the original publication. If only medians and ranges of the variables were reported, the missing mean values and SDs were generated statistically as described previously (8). If necessary, the mean values and ranges were calculated using the individual patient data from the original articles when possible. For longitudinal studies, the time point from which the most data could be gathered was chosen to represent the study. When mean binding ratios (BRs) were reported $(\mathrm{BR}=$ [region of interest]/reference), they were converted to specific binding ratios (SBRs) $(\mathrm{SBR}=([$ region of interest $]-$ reference $) /$ reference) by subtracting $1(\mathrm{SBR}=\mathrm{BR}-1)$.

The most common investigated brain regions were the mean (bilateral) caudate nucleus (25 studies), mean putamen (21 studies), mean striatum (15 studies), contralateral caudate nucleus (11 studies), ipsilateral caudate nucleus (11 studies), mean posterior putamen ( 9 studies), mean anterior putamen (8 studies), contralateral putamen (8 studies), and ipsilateral putamen (8 studies). Asymmetry indices for the putamen were reported in 6 studies, and putamen-to-caudate ratios were reported in 6 studies. For the AADC studies, sufficient data were available only for the mean caudate nucleus and mean putamen.

\section{Risk of Publication Bias and Quality of Studies}

The risk of publication bias was considered and examined using funnel plots of the studies included in the synthesis (comparisons with 10 or more samples). The quality of the included studies was evaluated according to the Newcastle-Ottawa scale (9).

\section{Statistical Analysis}

The study results and group comparisons were synthesized using Meta-Essentials (version 1.1; Erasmus Research Institute of Management) (10). Statistical significance was set at 2-tailed $P$ value of less than 0.05 . Hedges $g$ was used as an estimator of effect size in group comparisons using the random-effects model. Heterogeneity of the data was examined using 
TABLE 1

Summary of Demographic and Clinical Details of Samples in Included Studies

\begin{tabular}{|c|c|c|c|c|c|c|}
\hline Target & Variable & PD & MSA-P & PSP & CBS & MSA-C \\
\hline \multirow[t]{7}{*}{ DAT } & Samples $(n)$ & 26 & 18 & 16 & 5 & 5 \\
\hline & Patients (n) & 877 & 285 & 181 & 77 & 62 \\
\hline & Age (y) & $64(9)$ & $63(9)$ & $67(7)$ & $68(8)$ & $62(8)$ \\
\hline & Sex (M/F ratio) & 1.5 & 1.0 & 1.3 & 0.7 & 1.1 \\
\hline & Disease duration $(\mathrm{y})$ & $4.6(3.6)$ & $3.2(1.9)$ & $3.2(1.9)$ & $3.0(1.3)$ & $3.2(1.9)$ \\
\hline & Hoehn and Yahr scale & $2.1(0.7)$ & $3.4(0.8)$ & $3.2(0.9)$ & $3.0(0.8)$ & $3.5(1.1)$ \\
\hline & Motor UPDRS score & $23(11)$ & $37(13)$ & $33(11)$ & $35(13)$ & $30(11)$ \\
\hline \multirow[t]{7}{*}{ AADC } & Samples $(n)$ & 6 & 5 & 3 & 1 & 0 \\
\hline & Patients $(n)$ & 81 & 71 & 23 & 2 & 0 \\
\hline & Age $(y)$ & $58(6)$ & $57(7)$ & $66(5)$ & $65(5)$ & - \\
\hline & Sex (M/F ratio) & 2.1 & 1.6 & 3.3 & 1.0 & - \\
\hline & Disease duration $(\mathrm{y})$ & $8.3(6.3)$ & 4.7 (3.3) & $3.3(1.8)$ & $4.0(0)$ & - \\
\hline & Hoehn and Yahr scale & $2.7(0.8)$ & $3.3(0.8)$ & $3.3(-)$ & - & - \\
\hline & Motor UPDRS score & - & - & - & - & - \\
\hline
\end{tabular}

Numbers or weighted (for number of subjects for each study) mean values and weighted SDs (in parentheses) are presented.

$I^{2}$ statistics. If substantial heterogeneity $\left(I^{2}>50 \%\right)$ was detected, metaregression analyses of age, disease duration, and disease severity as indicated by the motor UPDRS and Hoehn and Yahr scale scores were also performed.

\section{RESULTS}

\section{Study Characteristics}

Twenty-nine DAT studies (Supplemental Table 1) and 6 AADC studies (Supplemental Table 2) were included in the metaanalysis (11-38) (supplemental materials are available at http://jnm.snmjournals. org). There were no suitable VMAT2 studies. Four studies (39-42) that had combined MSA-P patients with MSA-C patients were excluded from the MSA analysis (Supplemental Table 3). Only 1 study reported binding values also in PSP parkinsonism variant patients (43), which were not included in the analysis. The final sample thus included 35 studies that described DAT or AADC binding in 958 PD, 356 MSAP, 204 PSP, 79 CBS, and 62 MSA-C patients. The demographic and clinical characteristics of the patients are presented in Table 1. The quality evaluation of the included studies is presented in Supplemental Table 4. Twenty-five studies received 5-6 stars, and 10 studies received 3-4 stars (out of 6 stars) in the NewcastleOttawa scale. The overall quality of the studies was therefore sufficient, but the PET/SPECT imaging methodology and resolution were suboptimal in studies that had been published in the 1990s. There was some variation in diagnostic criteria as well (Supplemental Table 5), although many studies used published and commonly applied criteria for PD (44), PSP (45), and MSA (46,47).

The DAT studies were published between 1998 and 2018. The tracers used were ${ }^{123} \mathrm{I}-\beta$-CIT, ${ }^{123} \mathrm{I}$-FP-CIT, ${ }^{99 \mathrm{~m} T c-T R O D A T,}{ }^{18} \mathrm{~F}-\mathrm{FP}-$ CIT, and ${ }^{123} \mathrm{I}$-IPT. Most included studies had calculated striatumspecific binding ratios using the occipital cortex as the reference region, and the values were expressed as ([region of interest] [occipital cortex])/[occipital cortex]) (Supplemental Table 1). The AADC studies were published between 1990 and 1997 and used $6-{ }^{18}$ F-fluoro-L-dopa as the tracer.

\section{Atypical Parkinsonism Versus PD}

The PSP patients had lower DAT binding than did the PD patients in the mean caudate (weighted relative difference $=34.1 \%$, Table 2), mean putamen (18.2\%, Fig. 2, Table 2), mean striatum (Table 2), contralateral caudate, ipsilateral caudate, and anterior putamen (Supplemental Table 6). The MSA-P patients had lower DAT binding than did the PD patients in the mean caudate (Table 2). There were no differences between PD patients and patients with MSA-C or CBS, but the total numbers of patients and studies were low (Table 2). There were no differences in AADC activity between the PD and PSP or MSA-P patients, although directions of differences were similar to the DAT analysis (Supplemental Table 7). There were insufficient data for other AADC comparisons.

\section{Differences Between Types of Atypical Parkinsonism}

The PSP patients had $31.4 \%$ lower mean striatal DAT binding (weighted relative difference) than did the MSA-P patients (Fig. 2; Table 2). The MSA-P patients had $46.0 \%$ lower DAT binding than did the MSA-C patients in the striatum (Fig. 2; Table 2). No other significant differences were observed. The primary results with DAT imaging remained the same when only studies that had used similar diagnostic criteria (Supplemental Table 5) were included in the analysis. There were no suitable studies that had compared PSP with MSA-C patients, nor were there any that had compared CBS patients with those who had other types of atypical parkinsonism. There were no differences in AADC activity between PSP and MSA-P patients, and there were insufficient data for other comparisons (Supplemental Table 7).

\section{Metaregression Analyses and Publication Bias}

There were no significant associations in metaregression analyses using disease duration or the mean motor UPDRS values as moderators. The only significant relationship observed was between the Hoehn and Yahr scale and the effect size for caudate DAT binding in the PD versus MSA-P comparison (Supplemental Table 8), indicating that a higher 
difference in the Hoehn and Yahr scale scores between the PD and MSA-P groups was associated with a greater difference in caudate nucleus DAT binding between these groups. Funnel plots of the comparisons that had sufficient numbers of studies did not suggest missing studies that would have implied publication bias (Egger regressions, $P>0.05$ ).

\section{DISCUSSION}

The results of this metaanalysis indicated that striatal DAT binding is lower in PSP patients than in either MSA-P or PD patients. Another important finding was that caudate DAT binding is lower in MSA-P patients than in PD patients, without significant differences in the putamen. The third major finding was that striatal DAT binding is clearly lower in MSA-P patients than in MSA-C patients. Although not significant because of limited data, AADC results paralleled the findings with DAT. The data concerning VMAT2 and CBS are currently insufficient.

\section{Dopaminergic Function Is Lower in PSP Than in Either MSA-P or PD}

Our results show that presynaptic dopaminergic function, as measured by DAT binding, is up to 34\% lower in PSP than in MSA-P or PD. It has been demonstrated that there is a profound loss of nigral dopaminergic neurons in PSP (48), and on the basis of the present results, this loss may exceed that seen in other types of degenerative parkinsonism, at least when patients are examined by means of functional brain imaging 3-5 y after symptom onset. Comparative neuropathologic data are needed to investigate whether the greater loss of presynaptic dopamine function in PSP is present at all disease stages and whether this loss of dopamine function is based on greater neuronal loss or a functional difference in the nigrostriatal tract. There are data suggesting that PD and PSP patients may have similar losses of A9 dopamine neurons in the substantia nigra (49), whereas the number of A10 neurons is clearly lower in PSP than in PD (50). From a clinical perspective, it is important to note that the markedly lower DAT binding in the PSP patients compared with the PD or MSA-P patients does not seem to be directly related to clinical differences in motor symptom severity. For example, although the motor symptoms of the PSP patients were less advanced than those of the MSA-P patients (motor UPDRS score, 33 vs. 37, respectively), the striatal dopaminergic degeneration was clearly more progressed $31.4 \%$ lower in the PSP patients than in the MSA-P patients).

Relative differences in the striatal DAT binding between PSP and MSA-P/PD were large, at 18\%-34\% (Hedges $g>0.70$ ). The magnitudes are possibly diagnostically significant. Currently, many semiautomatic analysis systems used clinically for DAT SPECT have taken advantage of published cohorts of healthy subjects (e.g., Varrone et al. (51)), and clinical diagnostics is aided by the automatic flagging of abnormal striatal values as compared with the reference values. In the future, automated analysis could possibly be extended to atypical parkinsonism by including reference values for PD, PSP, MSA-P, and MSA-C. However, this would not be an easy task, as the pathologic level is not constant across the disease course, and the system would need to contain information about not only the age and sex of the patients but also the motor symptom severity and disease duration. Achieving this task may not be possible in the immediate future, but an endeavor toward this purpose could possibly be performed via the international collection 


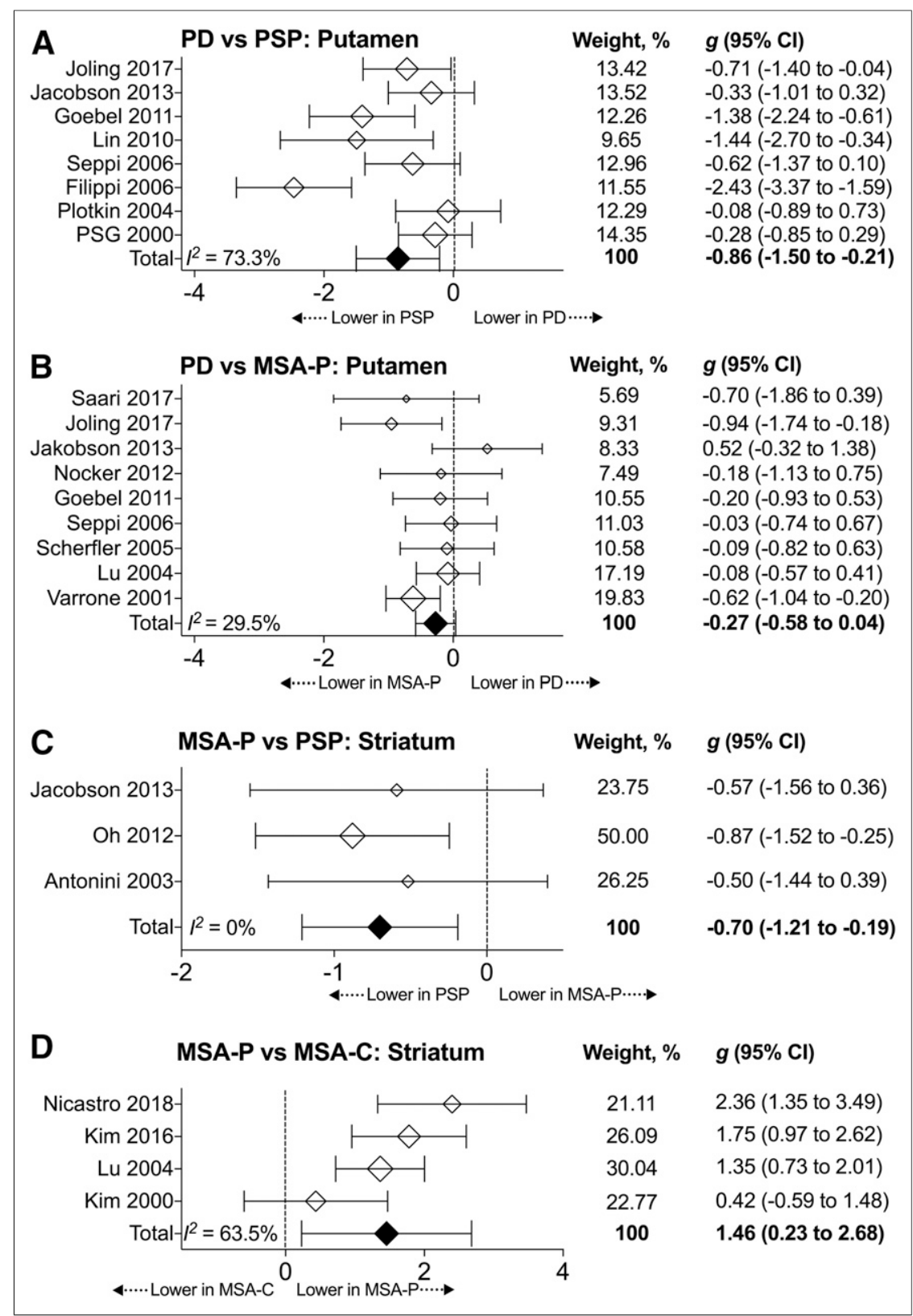

FIGURE 2. Forest plots of key comparisons in DAT studies. (A) Significant putaminal difference between PD and PSP. (B) Nonsignificant putaminal difference between PD and MSA-P. (C) Significant striatal difference between MSA-P and PSP. (D) Significant striatal difference between MSA-P and MSA-C. were variably reported. Nevertheless, the results indirectly support the notion that the caudate-to-putamen ratio may be affected in MSA. This is another issue that merits the further large multisite collection of clinical scans for comparison. An automated comparison of the caudate-to-putamen ratio to those from a large pool of clinically well-characterized patients with PD and atypical parkinsonism could prove valuable. These data would optimally be based on measurements from PET scans because of the superior spatial resolution, which allows clearer separation of striatal subregions than on SPECT scans (53). In the included studies, the binding values for the hemispheres contra- and ipsilateral to the predominant motor symptoms were also only sporadically reported. The lack of reported subregional and hemispheric values conveys a message to the neuroimaging community. To successfully perform similar metaanalyses in the future, more precise reporting of regional binding values (each contra- and ipsilateral region for each group together with SDs) or open data sharing is needed.

\section{MSA-P and MSA-C Differ in Striatal Dopamine Function}

There was a strikingly large $46.0 \%$ difference in striatal DAT binding between the MSA-P and MSA-C patients (Hedges $g=1.46,4$ studies with 133 patients). DAT imaging therefore appears useful in the differentiation of MSA subtypes. However, rather than being dichotomically different pathologic entities, MSA-P and MSA-C are likely to represent a neuropathologic continuum with mixed neuropathology (55). It is possible that MSA-P and MSA-C patients included in neuroimaging trials are particularly well characterized and represent extreme ends of the continuum. Therefore, the large difference in striatal DAT binding between the MSA-P and MSA-C patients possibly does not fully represent clinical reality, in which patients with mixed phenotypes are of large numbers of scans of patients with atypical parkinsonism (52).

\section{Caudate Dopaminergic Loss Differentiates MSA-P from PD}

The results also showed that while there does not seem to be a difference in putaminal dopaminergic function between MSA-P and $\mathrm{PD}$, there is a difference in the caudate nucleus. Indeed, one previously suggested possibility for improving the dopaminergic diagnostic accuracy of atypical parkinsonism is the use of caudateto-putamen or putamen-to-caudate ratios, as it has been suspected that the rostrocaudal gradient of the dopaminergic deficit is lost in atypical parkinsonism $(53,54)$. We were unable to perform metaanalytic calculations of these ratios because the measurements more frequent. Nevertheless, the magnitude of the difference is noteworthy, and we argue that striatal DAT imaging could be one of the auxiliary diagnostic tools for patients with mild parkinsonism, dysautonomic features, and variable levels of cerebellar findings. The second consensus diagnostic criteria of MSA suggested that in the absence of parkinsonian features in a patient with cerebellar ataxia, imaging evidence of a nigrostriatal presynaptic deficit points to the diagnosis of MSA-C (47). The present results do not directly contradict this interpretation, but the results demonstrate that it is not the MSA-C subtype but rather the MSA-P phenotype that shows the robust loss of dopamine function. Further studies comparing MSA-C with other degenerative parkinsonism will be important. 


\section{Limitations}

The results presented here were derived almost solely from DAT imaging using various tracers. We did not identify suitable VMAT2 studies, and the number of AADC studies was low (6 studies published in the 1990s). Therefore, we do not currently know if the diagnostic value of DAT imaging in atypical parkinsonism is superior to or worse than other presynaptic imaging targets. Although AADC function may be somewhat upregulated in PD and the DAT is possibly downregulated (56), we do not consider it likely that the differences reported here would be markedly different if VMAT2 or AADC were the target. Another limitation is that the level of the present evidence precludes definitive conclusions about the dopaminergic function in CBS because the numbers of studies and patients were low. It is also debatable whether it is useful to classify PSP and CBS as different disorders $(57,58)$. It should also be noted that the results of the present metaanalysis do not necessarily well represent clinical diagnostic reality, because many of the included studies were performed with patients who had already been clinically diagnosed at the time of imaging. Finally, medications were variably reported and it was therefore impossible to perform subanalyses between treatment groups.

\section{CONCLUSION}

The results of this metaanalysis demonstrate that PSP is associated with the greatest presynaptic dopaminergic loss compared with other degenerative parkinsonian syndromes. The observed large difference between MSA-P and MSA-C may also be clinically useful in patients with dysautonomia. Given the magnitude of the differences between the diagnostic groups, an effort could be initiated to collect and analyze clinical scans that could be used to create reference and cutoff values for research and clinical work.

\section{DISCLOSURE}

This research was supported by the Päivikki and Sakari Sohlberg Foundation and the Turku University Hospital (ERVA funds). Juho Joutsa was funded by the Academy of Finland (grant 295580). No other potential conflict of interest relevant to this article was reported.

\section{KEY POINTS}

QUESTION: It is unclear whether presynaptic dopamine imaging with PET or SPECT can be used in the differential diagnosis of atypical parkinsonism.

PERTINENT FINDINGS: Striatal DAT function was clearly lower in PSP than in PD or MSA-P and was clearly lower in MSA-P than in MSA-C.

IMPLICATIONS FOR PATIENT CARE: The results demonstrate group-level differences in presynaptic dopamine function between atypical parkinsonism syndromes.

\section{REFERENCES}

1. Kim HJ, Jeon BS, Jellinger KA. Diagnosis and differential diagnosis of MSA: boundary issues. J Neurol. 2015;262:1801-1813.

2. Joutsa J, Gardberg M, Röyttä M, Kaasinen V. Diagnostic accuracy of parkinsonism syndromes by general neurologists. Parkinsonism Relat Disord. 2014;20:840-844.
3. Hughes AJ, Daniel SE, Ben-Shlomo Y, Lees AJ. The accuracy of diagnosis of parkinsonian syndromes in a specialist movement disorder service. Brain. 2002;125: 861-870.

4. Kaasinen V, Vahlberg T. Striatal dopamine in Parkinson disease: a meta-analysis of imaging studies. Ann Neurol. 2017;82:873-882.

5. Strafella AP, Bohnen NI, Perlmutter JS, et al. Molecular imaging to track Parkinson's disease and atypical parkinsonisms: new imaging frontiers. Mov Disord. 2017;32:181-192.

6. Vlaar AM, van Kroonenburgh MJ, Kessels AG, Weber WE. Meta-analysis of the literature on diagnostic accuracy of SPECT in parkinsonian syndromes. BMC Neurol. 2007;7:27.

7. Moher D, Liberati A, Tetzlaff J, Altman DG, Group P. Preferred reporting items for systematic reviews and meta-analyses: the PRISMA statement. PLoS Med. 2009;6:e1000097.

8. Hozo SP, Djulbegovic B, Hozo I. Estimating the mean and variance from the median, range, and the size of a sample. BMC Med Res Methodol. 2005;5:13.

9. Wells G, Shea B, O'Connell D, et al. The Newcastle-Ottawa Scale (NOS) for assessing the quality of nonrandomized studies in meta-analyses. The Ottawa Hospital website. http://www.ohri.ca/programs/clinical_epidemiology/oxford.asp. Accessed June 21, 2019.

10. Suurmond R, van Rhee H, Hak T. Introduction, comparison, and validation of Meta-Essentials: a free and simple tool for meta-analysis. Res Synth Methods. 2017;8:537-553.

11. Messa C, Volonté MA, Fazio F, et al. Differential distribution of striatal [ $\left.{ }^{123} \mathrm{I}\right]$ betaCIT in Parkinson's disease and progressive supranuclear palsy, evaluated with single-photon emission tomography. Eur J Nucl Med. 1998;25:1270-1276.

12. Kim GM, Kim SE, Lee WY. Preclinical impairment of the striatal dopamine transporter system in sporadic olivopontocerebellar atrophy: studied with $\left[{ }^{123} I\right]$ betaCIT and SPECT. Eur Neurol. 2000;43:23-29.

13. Parkinson Study Group. A multicenter assessment of dopamine transporter imaging with DOPASCAN/SPECT in parkinsonism. Neurology. 2000;55:1540-1547.

14. Varrone A, Marek KL, Jennings D, Innis RB, Seibyl JP. $\left[{ }^{123}\right.$ I]beta-CIT SPECT imaging demonstrates reduced density of striatal dopamine transporters in Parkinson's disease and multiple system atrophy. Mov Disord. 2001;16:1023-1032.

15. Kim YJ, Ichise M, Ballinger JR, et al. Combination of dopamine transporter and $\mathrm{D}_{2}$ receptor SPECT in the diagnostic evaluation of PD, MSA, and PSP. Mov Disord. 2002; 17:303-312.

16. Antonini A, Benti R, De Notaris R, et al. ${ }^{123}$ I-ioflupane/SPECT binding to striatal dopamine transporter (DAT) uptake in patients with Parkinson's disease, multiple system atrophy, and progressive supranuclear palsy. Neurol Sci. 2003;24: 149-150.

17. Lai SC, Weng YH, Yen TC, et al. Imaging early-stage corticobasal degeneration with [ ${ }^{99 m}$ Tc]TRODAT-1 SPET. Nucl Med Commun. 2004;25:339-345.

18. Lu CS, Weng YH, Chen MC, et al. ${ }^{99 m}$ Tc-TRODAT-1 imaging of multiple system atrophy. J Nucl Med. 2004;45:49-55.

19. Scherfler C, Seppi K, Donnemiller E, et al. Voxel-wise analysis of $\left[{ }^{123}\right.$ I] beta-CIT SPECT differentiates the Parkinson variant of multiple system atrophy from idiopathic Parkinson's disease. Brain. 2005;128:1605-1612.

20. Swanson RL, Newberg AB, Acton PD, et al. Differences in [99m Tc]TRODAT-1 SPECT binding to dopamine transporters in patients with multiple system atrophy and Parkinson's disease. Eur J Nucl Med Mol Imaging. 2005;32:302-307.

21. Filippi L, Manni C, Pierantozzi M, et al. ${ }^{123}$ I-FP-CIT in progressive supranuclear palsy and in Parkinson's disease: a SPECT semiquantitative study. Nucl Med Commun. 2006;27:381-386.

22. Seppi K, Scherfler C, Donnemiller E, et al. Topography of dopamine transporter availability in progressive supranuclear palsy: a voxelwise $\left[{ }^{123} \mathrm{I}\right]$ beta-CIT SPECT analysis. Arch Neurol. 2006;63:1154-1160.

23. Roselli F, Pisciotta NM, Pennelli M, et al. Midbrain SERT in degenerative parkinsonisms: a ${ }^{123}$ I-FP-CIT SPECT study. Mov Disord. 2010;25:1853-1859.

24. Goebel G, Seppi K, Donnemiller E, et al. A novel computer-assisted image analysis of $\left[{ }^{123} \mathrm{I}\right] \beta$-CIT SPECT images improves the diagnostic accuracy of parkinsonian disorders. Eur J Nucl Med Mol Imaging. 2011;38:702-710.

25. Cilia R, Rossi C, Frosini D, et al. Dopamine transporter SPECT imaging in corticobasal syndrome. PLoS One. 2011;6:e18301.

26. Nocker M, Seppi K, Donnemiller E, et al. Progression of dopamine transporter decline in patients with the Parkinson variant of multiple system atrophy: a voxel-based analysis of $\left[{ }^{123} \mathrm{I}\right] \beta$-CIT SPECT. Eur J Nucl Med Mol Imaging. 2012;39:1012-1020.

27. Jakobson Mo S, Linder J, Forsgren L, Holmberg H, Larsson A, Riklund K. Preand postsynaptic dopamine SPECT in idiopathic Parkinsonian diseases: a followup study. BioMed Res Int. 2013;2013:143532.

28. Hammesfahr S, Antke C, Mamlins E, et al. FP-CIT- and IBZM-SPECT in corticobasal syndrome: results from a clinical follow-up study. Neurodegener Dis. 2016;16:342-347. 
29. Kim HW, Kim JS, Oh M, et al. Different loss of dopamine transporter according to subtype of multiple system atrophy. Eur J Nucl Med Mol Imaging. 2016; 43:517-525.

30. Joling M, Vriend C, van den Heuvel OA, et al. Analysis of extrastriatal ${ }^{123} \mathrm{I}$-FPCIT binding contributes to the differential diagnosis of parkinsonian diseases. J Nucl Med. 2017;58:1117-1123.

31. Ohta Y, Yamashita T, Hishikawa N, et al. Potential multisystem degeneration in Asidan patients. J Neurol Sci. 2017;373:216-222.

32. Saari L, Kivinen K, Gardberg M, Joutsa J, Noponen T, Kaasinen V. Dopamine transporter imaging does not predict the number of nigral neurons in Parkinson disease. Neurology. 2017;88:1461-1467.

33. Nicastro N, Garibotto V, Burkhard PR. ${ }^{123}$ I-FP-CIT SPECT accurately distinguishes parkinsonian from cerebellar variant of multiple system atrophy. Clin Nucl Med. 2018;43:e33-e36.

34. Brooks DJ, Salmon EP, Mathias CJ, et al. The relationship between locomotor disability, autonomic dysfunction, and the integrity of the striatal dopaminergic system in patients with multiple system atrophy, pure autonomic failure, and Parkinson's disease, studied with PET. Brain. 1990;113:1539-1552.

35. Brooks DJ, Ibanez V, Sawle GV, et al. Differing patterns of striatal ${ }^{18} \mathrm{~F}$-dopa uptake in Parkinson's disease, multiple system atrophy, and progressive supranuclear palsy. Ann Neurol. 1990;28:547-555.

36. Burn DJ, Sawle GV, Brooks DJ. Differential diagnosis of Parkinson's disease, multiple system atrophy, and Steele-Richardson-Olszewski syndrome: discriminant analysis of striatal ${ }^{18}$ F-dopa PET data. J Neurol Neurosurg Psychiatry. 1994;57:278-284.

37. Otsuka M, Ichiya Y, Kuwabara Y, et al. Nigrofrontal dopaminergic function as assessed by ${ }^{18}$ F-dopa PET. Nucl Med Commun. 1995;16:1021-1025.

38. Antonini A, Leenders KL, Vontobel P, et al. Complementary PET studies of striatal neuronal function in the differential diagnosis between multiple system atrophy and Parkinson's disease. Brain. 1997;120:2187-2195.

39. Otsuka M, Kuwabara Y, Ichiya Y, et al. Differentiating between multiple system atrophy and Parkinson's disease by positron emission tomography with ${ }^{18} \mathrm{~F}$-dopa and ${ }^{18}$ F-FDG. Ann Nucl Med. 1997;11:251-257.

40. Pirker W, Asenbaum S, Bencsits G, et al. [ $\left.{ }^{123} \mathrm{I}\right]$ beta-CIT SPECT in multiple system atrophy, progressive supranuclear palsy, and corticobasal degeneration. Mov Disord. 2000;15:1158-1167.

41. Berding G, Brücke T, Odin P, et al. [[ $\left.{ }^{123} I\right]$ beta-CIT SPECT imaging of dopamine and serotonin transporters in Parkinson's disease and multiple system atrophy. Nuklearmedizin. 2003;42:31-38.

42. Plotkin M, Amthauer H, Klaffke S, et al. Combined ${ }^{123}$ I-FP-CIT and ${ }^{123}$ I-IBZM SPECT for the diagnosis of parkinsonian syndromes: study on 72 patients. J Neural Transm. 2005;112:677-692.

43. Lin WY, Lin KJ, Weng YH, et al. Preliminary studies of differential impairments of the dopaminergic system in subtypes of progressive supranuclear palsy. Nucl Med Commun. 2010;31:974-980.
44. Hughes AJ, Daniel SE, Kilford L, Lees AJ. Accuracy of clinical diagnosis of idiopathic Parkinson's disease: a clinico-pathological study of 100 cases. J Neurol Neurosurg Psychiatry. 1992;55:181-184.

45. Litvan I, Agid Y, Calne D, et al. Clinical research criteria for the diagnosis of progressive supranuclear palsy (Steele-Richardson-Olszewski syndrome): report of the NINDS-SPSP international workshop. Neurology. 1996;47:1-9.

46. Gilman S, Low PA, Quinn N, et al. Consensus statement on the diagnosis of multiple system atrophy. J Neurol Sci. 1999;163:94-98.

47. Gilman S, Wenning GK, Low PA, et al. Second consensus statement on the diagnosis of multiple system atrophy. Neurology. 2008;71:670-676.

48. Williams DR, de Silva R, Paviour DC, et al. Characteristics of two distinct clinical phenotypes in pathologically proven progressive supranuclear palsy: Richardson's syndrome and PSP-parkinsonism. Brain. 2005;128:1247-1258.

49. Hardman CD, Halliday GM, McRitchie DA, Cartwright HR, Morris JG. Progressive supranuclear palsy affects both the substantia nigra pars compacta and reticulata. Exp Neurol. 1997;144:183-192.

50. Murphy KE, Karaconji T, Hardman CD, Halliday GM. Excessive dopamine neuron loss in progressive supranuclear palsy. Mov Disord. 2008;23:607-610.

51. Varrone A, Dickson JC, Tossici-Bolt L, et al. European multicentre database of healthy controls for [ ${ }^{123}$ I]FP-CIT SPECT (ENC-DAT): age-related effects, gender differences and evaluation of different methods of analysis. Eur J Nucl Med Mol Imaging. 2013;40:213-227.

52. ASAP SYNTAU: alignment and standardization of neuroimaging methods in atypical parkinsonism, specifically synucleinopathies and tauopathies-report of a JPND working group on harmonisation and alignment in brain imaging methods. JPND Research website. http://www.neurodegenerationresearch.eu/ wp-content/uploads/2018/04/JPND-Brain-Imaging-Working-Group-Report_ASAPSynTau.pdf. Published April 2018. Accessed June 21, 2019.

53. Oh M, Kim JS, Kim JY, et al. Subregional patterns of preferential striatal dopamine transporter loss differ in Parkinson disease, progressive supranuclear palsy, and multiple-system atrophy. J Nucl Med. 2012;53:399-406.

54. Im JH, Chung SJ, Kim JS, Lee MC. Differential patterns of dopamine transporter loss in the basal ganglia of progressive supranuclear palsy and Parkinson's disease: analysis with [ $\left.{ }^{123} \mathrm{I}\right] \mathrm{IPT}$ single photon emission computed tomography. J Neurol Sci. 2006;244:103-109.

55. Jellinger KA. Neuropathology of multiple system atrophy: new thoughts about pathogenesis. Mov Disord. 2014;29:1720-1741.

56. Lee CS, Samii A, Sossi V, et al. In vivo positron emission tomographic evidence for compensatory changes in presynaptic dopaminergic nerve terminals in Parkinson's disease. Ann Neurol. 2000;47:493-503.

57. Ling H, Macerollo A. Is it useful to classify PSP and CBD as different disorders? Yes. Mov Disord Clin Pract (Hoboken). 2018;5:145-148.

58. Lang AE. Comment on "Is it Useful to Classify PSP and CBD as Different Disorders?" Mov Disord Clin Pract (Hoboken). 2018;5:564-565. 